\title{
Pharmacy, Drug Development and Technology: from the Laboratory to Pharmacy
}

\author{
Nagba Yendoubé Gbandjaba* \\ Laboratoire de Chimie Organique et des Substances Naturelles, Université de Lomé, Lomé, Togo \\ *Corresponding Author: Nagba Yendoubé Gbandjaba, Laboratoire de Chimie Organique et des Substances Naturelles, Université de \\ Lomé, Lomé, Togo.
}

Received: July 05, 2019; Published: July 18, 2019

DOI: $10.31080 /$ ASPS.2019.03.0345

The discovery and development of a novel therapeutic agent to sell in the Pharmacy require a huge investment of time, money and the process is very risky [1-3]. The cost of drug development includes the discovery, the clinical trials and the failed drug fees [4].

Generally, the discovery time is situated between 3-20 years and is very essential because researchers spend all their time to investigate the property and the structure of the molecule that can fight the illness $[2,5]$.

After this step, researchers start the preclinical trial which consists in working on animals. This phase gives immense details about the pharmacology, the pharmacokinetics and the toxicity of the testing drugs. The next step is the clinical trial from phase I to IV focuses on human subjects [6].

Note that, we have now in the literature the phase 0 trial which is a platform for drug development [7]. Phase 0 trial is a recent designation for optional exploratory trials conducted. Phase 0 trial means the administration of single subtherapeutic doses of the study drug to a small number of subjects around 10 to $15[7,8]$. By addition there, no data concerning the safety and the efficacy of the drug is obtained. This step is simply made to investigate the best pharmacokinetic parameters in humans [8]. It just prepares the phase I trial.

In the phase I trial, a small healthy volunteers is recruited between 2 to 200 participants. Researchers test the safety, the side effects, the best dose, and the formulation method for the drug [9]. The study is conducted generally in a clinical trial clinic, where the subject can be observed by full-time staff and Physicians [9]. The volunteers are also paid a variable inconvenience fee for their time spent by the sponsor. A phase II trial is made to check the biological effect of the drug [10]. For the phase II trial, 100 to 300 participants are recruited to follow how well the drug works.
Phase III trial is aimed to investigate how well the drug is by comparison to a "gold standard" treatment. Around 300 to 3000 participants are recruited. Researcher said that, this step is the most expensive for them because it is long and time consuming [2]. The phase III is also called the "premarketing phase" because it mesures the consumer response to the drug.

When the drug has proved satisfactory after Phase III trials, sponsors combine all the information about the methods, the results of human and animal studies, manufacturing procedures, formulation details and shelf life in a documents.

The phase IV is the postmarketing surveillance trial. Sponsoring Company checked a new market for their drug. The phase IV trial involves pharmacovigilance in which the safety surveillance of the drug is made to detect any rare or long-term adverse effects over a much larger patient population and longer time period than was possible during the Phase I-III clinical trials. For the phase IV clinical trial the minimum time period mandatory is 2 years.

In the world, approximatively 7935 new drugs are in development in 2017. It is true, failed drug exists because industry estimates that on 10000 molecules screened in the discovery process, only one will make it to the market as approved drug. Industry will never recuperate the cost of failed drugs [2].

An marketing authorization is issued to maintain the pharmaceutical product on the market. One of the raison why marketing authorization may be withdrawn, suspended, revoked is that, under normal condition of use the benefit over risk ratio is no more favorable or the drug is lack of therapeutic efficacy. This is why some drugs are retracted in the pharmacy after marketing authorization issued. 


\section{Bibliography}

1. Dickson M and Gagnon JP. "The cost of new drug discovery and development". Discovery Medicine 4 (2004): 172-179.

2. Tonkens R. "An overview of the drug development process". Physician Executive 31 (2005): 48-52.

3. Dimasi JA. "Risks in new drug development: approval success rates for investigation all drugs". Clinical Pharmacololgy and Therapeutics 69 (2001): 297-307.

4. Rawlins MD. "Cutting the cost of drug development?" Nature Review Drug Discovery 3 (2004): 360-364.

5. Jacobson-Kram D and Mills G. "Leveraging exploratory investigational new drug studies to accelerate drug development". Clinical Cancer Research 14 (2008): 3670-3674.

6. Sedgwick P. "What are the four phases of clinical research trials?" BMJ 348 (2014): g3727.

7. Twombly R. "Slow start to phase 0 as researchers debate value". Journal of the National Cancer Institute 98 (2006): 804806.

8. Doroshow JH and Parchment RE. "Oncologic phase 0 trials incorporating clinical pharmacodynamics: from concept to patient". Clinical Cancer Research 14 (2008): 3658-3663.

9. Calvert AH and Plummer R. "The development of phase I cancer trial methodologies: the use of pharmacokinetic and pharmacodynamic end points sets the scene for phase 0 cancer clinical trials". Clinical Cancer Research 14 (2008): 3664-3669.

10. Booth CM., et al. "Design and conduct of phase II studies of targeted anticancer therapy: recommendations from the task force on methodology for the development of innovative cancer therapies (MDICT)". European Journal of Cancer 44 (2008): 25-29.

Volume 3 Issue 8 August 2019

(c) All rights are reserved by Nagba Yendoubé

Gbandjaba.

Citation: Nagba Yendoubé Gbandjaba. "Pharmacy, Drug Development and Technology: from the Laboratory to Pharmacy". Acta Scientific Pharmaceutical Sciences 3.8 (2019): 96-97. 